\title{
diálogo con gert biesta: filosofía y educación
}

\author{
félix garcía moriyón ${ }^{1}$ \\ universidad autónoma de madrid, españa
}

resumen

Biesta plantea, entre otros, varios temas que merecen algunas precisiones. En primer lugar, destaca que la subjetivación debe ocupar un lugar preferente en la educación, tesis con la que estoy de acuerdo, pero también son fundamentales, de manera especial en la educación formal, las otras dos funciones, la profesionalización y la socialización. El objetivo, por tanto, es lograr un adecuado equilibrio entre las tres que haga posible un pleno proceso educativo. Por otra parte, bien está recuperar el valor del acto de educar en un momento en el que se pone mucho énfasis en el aprendizaje. Sin embargo, es igualmente necesario encontrar un equilibrio entre aprendizaje y educación, por lo que no se debe resaltar una contraposición entre ambas dimensiones del proceso educativo: no hay educación sin aprendizaje, no hay aprendizaje sin educación. Del mismo modo, la evaluación y la medición son elementos imprescindibles de la educación, aunque impliquen riesgos que deben ser evitados. Si nos planteamos algunos objetivos cuando educamos, tenemos que ser capaces de averiguar si los hemos alcanzado y en qué grado lo hemos hecho. Es decir, hay que medir y comparar. Por último, la educación es una relación interpersonal caracterizada por la apertura y el encuentro, que incluyen una visión positiva de la heteronomía y de la obediencia, en la que el empoderamiento no se opone a la aceptación de la interdependencia y la vulnerabilidad. Es una idea importante y bella que merece ser explorada en profundidad.

palabras clave: subjetivación; aprendizaje; enseñanza; evaluación; medida; relación interpersonal

\section{dialogue with gert biesta: philosophy and education}

abstract

Biesta approaches different important educational topics that deserve some clarification and exploration. To begin with, Biesta emphasizes the preferential position that subjectification should occupy in education, a thesis I agree with. Nevertheless, qualification and socialization are also fundamental domains in education, much more in formal education. The aim of education, therefore, is to achieve an adequate balance between the three domains that makes possible a full educational process. On the other hand, it is valuable proposal to recover the importance of the act of teaching, in a period of learning focuses the attention in educational environments. However, we need also to achieve a balance between teaching and learning; thus, a contrast between the two dimensions of education should not be highlighted: there is not teaching without learning, and there is not learning without teaching. Likewise, evaluation and measurement are essential elements of education, even if they involve risks that must be avoided. If we want to achieve some educational aims, we have to be able to find out whether we got them and how much we have achieved. That is, we have to measure and compare. Finally, education is an interpersonal relationship characterized by openness and

\footnotetext{
1 E-mail: felix.garciamoriyon@gmail.com
} 
encounter, which includes a positive view of heteronomy and obedience, in which empowerment does not preclude acceptance of interdependence and vulnerability.

keywords: subjectivation; learning; teaching; assessment; measurement; interpersonal relationship.

\section{diálogo com gert biesta: filosofia e educação}

resumo

Biesta levanta, entre outros, vários assuntos que merecem algumas precisões. Em primeiro lugar, ele enfatiza que a subjetivação deve ocupar um lugar preferencial na educação, uma tese com a qual concordo, mas também são fundamentais, especialmente na educação formal, as outras duas funções, profissionalização e socialização. O objetivo, portanto, é alcançar um equilíbrio adequado entre os três que possibilitem um processo educacional completo. Por outro lado, é bom recuperar o valor do ato de educar em um momento em que muita ênfase é colocada na aprendizagem. No entanto, também é necessário encontrar um equilíbrio entre aprendizagem e educação, por isso não é necessário destacar o contraste entre as duas dimensões do processo educacional: não há educação sem aprender, não há aprendizado sem educação. Do mesmo modo, a avaliação e a medição são elementos essenciais da educação, mesmo que envolvam riscos que devem ser evitados. Se elevarmos alguns objetivos quando educamos, devemos descobrir se os conseguimos e até que ponto. Ou seja, é preciso medir e comparar. Finalmente, a educação é uma relação interpessoal caracterizada por abertura e encontro, que inclui uma visão positiva da heteronomia e obediência, na qual o empoderamento não impede a aceitação de interdependência e vulnerabilidade. É uma ideia importante e linda que merece ser explorada em profundidade.

palavras-chave: subjetivação; aprendizagem; ensino; avaliação; medição; relacionamento interpessoal. 
diálogo con gert biesta: filosofía y educación

Varios puntos llaman mi atención en la escucha atenta de la conferencia de Biesta, apoyado en una presentación escrita que resume su conferencia y en algunos de sus trabajos previos, en especial en el último de ellos, recién publicado en español, El hermoso riesgo de educar (BIESTA, 2017). Mi punto de partida es una sintonía sustancial con lo que el autor propone. No obstante, hay dos tesis defendidas por Biesta que merecen una especial reflexión y una tercera tesis que merecería una exploración más detallada para ampliar el alcance de todo lo que está proponiendo.

\section{la subjetivación como objetivo prioritario de la educación}

La primera tesis discutible es su insistencia en una cierta contraposición entre las tendencias dominantes en la educación actual y las que debieran ser dominantes. En este caso vamos a hablar solo de la educación formal, dejando al margen tanto la no formal como la informal. Podemos aceptar las tres funciones de la educación formal que Biesta señala: profesionalización, socialización y subjetivación. Él insiste en que se da prioridad a las dos primeras, quizá más a la primera de todas, y se margina la tercera, que sería la fundamental y la que debería tener un protagonismo más claro. Es una tesis que goza de gran predicamento entre las personas dedicadas a la enseñanza de la filosofía, en la medida en que consideran que hay un deterioro de la misma relacionado precisamente con un deterioro generalizado de la centralidad de la formación de sujetos plenos. Marta Nussbaum (2010) sería una representante cualificada de este tendencia crítica.

Puede haber bastante razón, siempre y cuando no se lleve demasiado lejos la crítica. Como el mismo Biesta afirma, lo importante es mantener un equilibrio entre las tres, de tal modo que ninguna anule las otras, aunque considero que la subjetivación debe ocupar un lugar jerárquicamente superior. La implantación de la educación formal obligatoria durante la infancia y la adolescencia es algo que comenzó a generalizarse a comienzos del siglo XIX en países del ámbito cultural 
occidental, para extenderse posteriormente a todo el mundo hasta llegar a ser universal en estos momentos, con limitaciones significativas en algunos países y más todavía en el caso de las niñas en determinadas zonas de la Tierra. Y sin entrar en la calidad de los sistemas educativos: son solo las cifras que ofrece el IDH.

Dos factores decisivos de este imparable crecimiento guardan directa relación con los procesos de profesionalización y socialización. El inicio de la primera revolución industrial exigía una formación profesional que excedía el modelo previo de los gremios; del mismo modo, el incremento de la población urbana requería procesos de socialización que garantizaran la integración de las nuevas generaciones en sociedades más complejas, algo que excedía las capacidades del núcleo familiar y era difícil en sociedades cada vez más urbanas; según la ONU, el 54\% de la población vive en áreas urbanas (NACIONES UNIDAS, 2014). Profesionalizar y socializar son, por tanto, rasgos constitutivos de todo sistema educativo, o para ser más precisos, del proceso de escolarización obligatoria. Junto a esos dos, y más sobre todo a partir del reconocimiento creciente del valor de la infancia como etapa de la vida intrínsecamente valiosa no subordinada a la etapa futura de la vida adulta, está siempre presente en la educación formal el objetivo del pleno desarrollo o crecimiento de la persona concreta e individual, como consta en documentos oficiales de la UE, la OCDE y en casi todos los países, como es el caso de España. Un documento importante que deja claro esta primacía jerárquica de la educación como proceso de subjetivación es el de Edgar Faure (1973), Aprender a ser que retoma el informe dirigido por Delors (1996), en el que se insiste en la importancia de incrementar la educación formal obligatoria y mejorar su calidad precisamente para conseguir esa formación completa que daría coherencia y sentido a los esfuerzos educativos de los gobiernos. Son, por tanto, cuatro los pilares que sustentan una educación de calidad: conocer; hacer; participar y cooperar; ser. En estos momentos, la escuela formal obligatoria manifiesta un compromiso explícito con la consolidación de sociedades democráticas 
La escuela obligatoria, por tanto, se hace cargo de lo que suele recibir el nombre de formación o iniciación a la vida adulta, de la Bildung (FABRÉ, 2008; HORLACHE, 2011), un concepto que, por otra parte posee una flexibilidad y una ambigüedad de significado muy similar al de subjetivación empleado por Biesta (HORLACHE, 2014). El objetivo de lograr esa formación global no está exento de contradicciones y tampoco está libre de una agenda oculta, es decir, de objetivos que, sin estar explícitamente expresados, son los que de hecho tienen una incidencia considerable en las personas y en la sociedad. Carlos Lerena lo expresaba bien en el título de su amplio ensayo sobre la historia de la educación occidental Reprimir y Liberar (LERENA, 2005): toda la educación occidental está marcada por tendencias casi opuestas, una socialización entendida como troquel de las conciencias (GARCÍA MORIYÓN, 2011) frente a otra que busca el crecimiento personal liberador.

El problema central de la educación ha sido, y sigue siendo, no tanto cuál es el específico orden jerárquico de esas tres dimensiones, algo que varía en las diversas etapas educativas y en diversos ámbitos de educación formal, sino más bien cuál es la específica configuración de cada una de ellas y cómo se articula la relación entre las tres. Retomando una importante distinción de Biesta, lo difícil es ser capaces de distinguir entre lo que se desea lograr en cada una de esas tres dimensiones y lo que sería deseable dados los problemas y retos específicos de cada contexto educativo y en cada época histórica.

\section{aprendificación (learnification) y mensurabilidad}

Biesta denuncia con fuerza la learnification, esto es el giro hacia una enseñanza centrada en el niño, para la que lo importante es el aprendizaje, no la educación. Este giro, que en su momento se planteó como un genuino giro copernicano, termina diluyendo el proceso educativo que no consiste tanto ni solo en lo que el niño aprende, sino también en lo que el profesorado logra enseñar al alumnado. Sin olvidar las conexiones que, según Biesta, este giro tiene con el pragmatismo y con el constructivismo, hay dos aspectos del mismo que inciden negativamente en el acto de educar. Por un lado, se convierte al profesorado en 
facilitador del aprendizaje, lo que socava su papel activo en el proceso de educación de la infancia y la adolescencia. Por otro lado, se exige precisar objetivos de aprendizaje, entendidos como competencias y/o destrezas, que deben estar bien definidas y, sobre todo, ser mensurables.

Según la crítica de Biesta, en los actuales sistemas educativos, ya no hay maestros, sino facilitadores; ya no hay escuelas o aulas, sino contextos o espacios de aprendizaje. Desde luego, en la medida en que esto es cierto, y en parte lo es, se está provocando una cierta distorsión de la situación educativa al pasar por alto dimensiones fundamentales de la misma. Parece, por tanto, necesario reivindicar y recuperar un papel más activo del profesorado, pero debemos cuidar que eso no contribuya a promover modelos magisteriales, al decir de los antiguos, o bancarios, al decir de Freire. Obviamente, no es eso lo que propone Biesta y conviene no olvidarlo, pero también es bueno recordar que la enseñanza magisterial tiene importantes carencias.

La relación pedagógica es una relación interpersonal asimétrica: hay un adulto que tiene la responsabilidad de proporcionar algo más que un contexto de aprendizaje para que la persona en proceso de ser educada pueda aprender adecuadamente. El profesorado debe tener un papel más activo en el sentido de dotar al proceso educativo tanto de contenidos como de intenciones. Paulo Freire es un buen ejemplo de una forma de entender la educación como actividad en la que el profesorado toma partido, en su caso en favor del oprimido, de la liberación y la concienciación. Maestras y maestras tienen, por tanto, la responsabilidad de tener claro qué tipo de profesionalización, de socialización y de subjetivación quieren promover en el aula. Y esa decisión es personal e intransferible, lo que exige, como bien señala Biesta, sabiduría práctica (prudencia) para saber tomar las decisiones adecuadas.

No es una decisión arbitraria, pues tiene que tener en cuenta que los grandes objetivos educativos se definen tras un debate político en el que participa toda la sociedad, después de un riguroso proceso deliberativo, tal y como se plantea en sociedades en las que funciona un modelo de democracia 
representativa. Tiene que tener en cuenta también el contexto más delimitado de su escuela o centro educativo, porque en ese ámbito se concretan los proyectos educativos con la implicación de toda la comunidad educativa, lo que incluye tanto al profesorado como a las familias y a los propios alumnos. Por último, debe tener en cuenta al alumnado, puesto que, solo con la implicación activa de los alumnos, se completa la relación interpersonal educativa: alguien enseña y alguien aprende. Si quien debe aprender no quiere hacerlo, por razones bien diversas, no se da el acto educativo; o se da, pero aprende algo diferente a lo que el educador plantea. De ahí que el propio Biesta hable de la educación como riesgo: la tarea educativa siempre puede fracasar, o quedarse lejos de sus objetivos. El éxito de la tarea docente no está nunca ciento por ciento garantizado; es un ámbito en el que dominan lo imprevisible y lo impredecible.

Esto último nos lleva al segundo aspecto de esta apartado: no hay educación sin evaluación del proceso educativo. Y no se puede evaluar si no se mide. En toda la educación formal existen calificaciones escolares en las que se mide el logro del alumnado y, lógicamente, ese logro permite establecer comparaciones. Educar exige medir, incluso cuando el objetivo es la subjetivación, entendida por Biesta como un modo no-egocéntrico de ser en el mundo y no como un proceso de desarrollo. Si el objetivo es pasar de un estado infantil a otro adulto, al final tendremos que saber con cierta precisión de qué estamos hablando cuando hablamos de adultez (grow-upness), y luego tendremos que saber si se ha logrado el objetivo y en qué medida se ha logrado. Lo que a su vez, nos obligará a revisar lo que hemos hecho en el aula para introducir modificaciones que mejoren esa práctica docente.

Existen críticas muy duras a todo lo relacionado con las competencias, de las que Biesta se hace amplio eco, y también de los esfuerzos realizados para medir el dominio de esas competencias, que han adquirido un notable protagonismo en la educación actual. Las críticas arrecian cuando se habla de PISA, el buque insignia de esa insistencia en definir objetivos mensurables y medirlos (CARABAÑA, 2015). Esas críticas son necesarias, pero no invalidan el 
objetivo de fondo. El problema es que hay que evaluar bien, teniendo claros cuáles son los objetivos de la evaluación y diseñando procedimientos válidos y fiables de evaluación (GARCÍA MORIYÓN, 2002). Es oportuno a este respecto recordar, por ejemplo, la gran tarea realizada por Amartya Sen y otros autores para lograr una evaluación válida y fiable de objetivos ambiciosos como son el desarrollo humano (MOLINA-PASCUAL, 2014).

No es cierto, por tanto, que todo lo verdaderamente importante en educación no se puede medir ni que medir equivale a etiquetar, clasificar y excluir-incluir a los estudiantes, aunque pueda ser utilizado para ello. La estrecha relación entre competencias, evaluación y contenidos educativos pretende, más allá de todas las críticas bien justificadas, aportar rigor al proceso educativo mediante un buen modelo de evaluación. Se cumple así con una doble exigencia: una rendición de cuentas que democráticamente justifique el enorme gasto educativo; una verificación empírica válida y fiable de que lo que decimos que intentamos hacer en los centros educativos realmente lo estamos haciendo.

\section{la educación como encuentro con el otro}

Biesta hace una afirmación discutible: el programa FpN (P4C) busca formar personas que planteen preguntas y cuestionen, pero no insiste tanto en cuestionar a esas mismas personas. Es decir, no cuestiona la identidad personal de quien pregunta. No comparto esta tesis. Más bien al contrario, el programa invita a los estudiantes a reflexionar sobre los propios pensamientos para someterlos a cuestionamiento permanente; ese es el sentido profundo del último capítulo de $E l$ descubrimiento de Harry (LIPMAN, 1990) o del episodio de la cadena y el cable en Lisa (LIPMAN, 1992). Aporta al proceso educativo un rasgo específico de la filosofía occidental desde Sócrates: actuar como el pez torpedo cuestionando las creencias personales. Por eso, no se trata de que el alumno haga preguntas, sino que haga "buenas" preguntas y se trata igualmente de que aporte respuestas tentativas bien argumentadas. La profesora debe, con las segundas preguntas dirigidas a las respuestas iniciales del estudiante, cuestionarlo y obligarlo a fundamentar lo que está afirmando. Es un constante poner en cuestión lo que 
dicen, que algunos, como Brenifier (2005), acentúan hasta convertirlo en un proceso catártico de puesta en cuestión de la propia identidad, lo que les aleja del modelo compartido en FpN.

Por eso mismo, en sintonía con Biesta, la comunidad de investigación filosófica no promueve estrategias inteligentes de adaptación al mundo entorno, sino que pone al alumnado y al profesorado frente a un mundo que les interpela, como diría Levinas, o que se presenta con la poderosidad de lo real que demanda de las personas cierto nivel de obediencia, como diría Zubiri (2012).

El punto de partida del proceso educativo es un sujeto previo, el niño filósofo, que hace preguntas y dota de sentido a sí mismo y al mundo en el que está; pero es también un sujeto que, desde el nacimiento, está abierto a un amplio abanico de posibilidades que tiene que ir definiendo. Es además un ser que nace en deuda, con deberes, frente a quienes le hacen vivir con solicitud y cuidado. El nacimiento nos sitúa en un mundo que está ahí, que se nos impone con forzosidad, del mismo modo estamos forzados a ser nosotros mismos y no otras personas. La religación nos sitúa precisamente ante el poder de lo real que se apodera de nosotros. Solo si admitimos ese hecho y sacamos las debidas consecuencias (lo que significa un sometimiento aceptado y reflexivo a ese poder) estamos en condiciones de asumir esa forzosidad de ser nosotros mismos que se nos impone como tarea. Ese apoderamiento es, por tanto y siguiendo con Zubiri, fundamento último, pero también es posibilitante (nos da las condiciones para poder ser) e impelente (nos fuerza a ser).

En la comunidad de investigación, el sujeto se siente interpelado por el mundo y por el otro. Un sujeto que se abre al acontecimiento en el que el otro se me presenta y me cuestiona, me despierta, un sujeto que es en primer lugar responsabilidad (BAUMAN, 2003), lo que hace de la ética el pilar de la vida personal y de la reflexión filosófica. Las relaciones entre autonomía y heteronomía, entre dependencia e independencia, no son relaciones dicotómicas que presentan disyunciones exclusivas. La heteronomía, el origen de la norma fuera de nosotros, en el mundo y en los otros sujetos, forma parte de nuestra vida, y demanda de 
nosotros una obediencia que no nos aliena, sino que precisamente nos hace libres, pues tenemos que hacer lo que ninguna otra persona podría hacer por nosotros. Del mismo modo, la legítima aspiración a la independencia que conlleva el deseo de ser una persona adulta, no está nunca separada de la constitutiva dependencia del ser humano: sin el mundo, sin los otros, como son, no como nos gustaría que fueran, no es posible llegar a ser la persona que somos y queremos ser ni llegar a vivir en el mundo en el que queremos vivir.

Educar es llamar a existir en el mundo, un mundo que no es construcción, sino que opone resistencias; la madurez significa permanecer en un término medio entre destruir un mundo que se resiste o destruir el propio yo vencido por esa resistencia. El diálogo no es ya un conversación sino una forma existencial, un desafío de por vida. Implica una escucha inteligente y abierta de lo que la otra persona nos dice, lo que en el fondo exige apertura al otro que siempre nos interpela y que no se debe manipular. Es una reivindicación de la hospitalidad como apertura radical al otro, hospitalidad que permite acceder a una subjetividad no enclaustrada en una empobrecedora auto-referencialidad solipsista. Es un enfoque desarrollado por Derrida, interpretando la posición de Levinas, como bien indica Fernández Agis (2009).

Estas reflexiones no son sino una aportación para enriquecer lo que, en definitiva, constituye una idea central de Biesta: la educación tiene que ver con llegar a ser un adulto en el mundo que descentra su punto de vista, que deja de ser el centro del universo y revisa si sus deseos son realmente deseables. Por eso mismo, la educación implica: a) interrupción de los deseos y el egocentrismo; b) suspensión, creando espacios y tiempos para poder encontrar y cuestionar los propios deseos; c) apoyo o sostén para mantenerse en ese punto medio entre el deseo y lo deseable. Desde luego esto implica fortalecer la dimensión cognitiva, empoderar, pero también, romper las resistencias, desarmar un ego siempre dispuesto a sobreactuar con estrategias de imposición y dominio, falso camino que lejos crecer a partir del poder impelente de nuestra intrínseca vulnerabilidad y 
dependencia, convierte a ambas en factores de empobrecimiento personal y debilitamiento.

\section{referencias}

CARABAÑA, Julio. La inutilidad de PISA para las escuelas. Madrid: Catarata, 2015. BAUMAN, Z. Modernidad líquida. México: FCE, 2003.

BRENIFIER, Oscar. El diálogo filosófico. Santa Cruz de Tenerife: Idea, 2005.

DELORS, Jacques. La educación encierra un tesoro. Informe a la UNESCO sobre la educación para el siglo XXI. Madrid: Santillana/UNESCO, 1996.

FABRE, Michel. "Expérience et formation, la Bildung", en Penser la formation, París, Presses Universitaires de France., 2008.

FAURE, Edgar. Aprender a ser. El mundo de la educación hoy y mañana. Madrid: UNESCO-Alianza, 1973.

FERNÁNDEZ AGIS, D. Tiempo, política y hospitalidad. Una reflexión desde Derrida y Lévinas. Isegoría. Revista de Filosofía Moral y Política. 40, p. 191-202, 2009.

GARCÍA MORIYÓN, Félix. (Coord.). La estimulación de la inteligencia cognitiva y la inteligencia afectiva. Madrid: De la Torre, 2002.

GARCÍA MORIYÓN, Félix. El troquel de las conciencias. Una historia de la educación moral en España. Madrid: De la Torre, 2011.

HORLACHE, Rebekka. ¿Qué es Bildung? El eterno atractivo de un concepto difuso en la teoría de la educación alemana. Pensamiento Educativo. Revista de Investigación Educacional Latinoamericana, 51(1), p. 35-45, 2014.

HORLACHE, Rebekka. Bildung, la formación. Barcelona: Octaedro, 2011.

LERENA ALESÓN, Carlos. Reprimir y liberar. Crítica sociológica de la educación y la cultura contemporánea. Tres Cantos (Madrid): Akal, 2005.

LIPMAN, Matthew. El descubrimiento de Ari Stóteles. Madrid: De la Torre, 1990.

LIPMAN, Matthew. Lisa. Madrid: De la Torre, 1992.

NACIONES UNIDAS. La situación demográfica en el mundo, 2014. Informe conciso. New York: Naciones Unidas, 2014.

MOLINA SALAZAR, R.E.; PASCUAL GARCÍA, J.M. El Índice de Desarrollo Humano como indicador social, en Nómadas. Revista Crítica de Ciencias Sociales y Jurídicas, vol. 44, 4, 2014.

NUSSBAUM, Martha. Non for profit. Why Democracy needs the Humanities. Princeton: Princeton Univ. Press, 2010.

ZUBIRI, X. El hombre y Dios. Madrid: Alianza, 2012.

recibido en: 11.08 .2017

aprobado en: 31.08 .2017 